\title{
Comparison of five methods for the determination of arginine hydrolysis by viridans streptococci
}

\author{
P. W. J. WEST, H. A. FOSTER*, QUDSIYA ELECTRICWALA and ALEYAMMA ALEX \\ Department of Medical Laboratory Technology, Kuwait University, 90805 Suleibikhat, Kuwait and *Department \\ of Biological Sciences, University of Salford, Salford M5 4WT
}

\begin{abstract}
Seventy-eight clinical isolates and four control strains of viridans streptococci were tested in parallel for arginine hydrolysis by five different methods. These comprised two commercial systems, the API20 STREP and Vitek GPI card, two published methods, one based on ammonia production and one on alkalisation of Moeller's decarboxylase medium, and a method based on alkalisation of a phenol-red broth medium dispensed into microtitration plates. The clinical isolates were speciated by their biochemical reactions in the API20 STREP and API20 ZYM systems. One strain produced only a weak reaction for arginine hydrolysis in the medium by ammonia production, but otherwise the results with this medium, the API20 STREP and the microtitration plate method were identical. Tests with the Møeller decarboxylase medium and Vitek GPI card gave negative results with isolates that were positive by other methods. Inoculum size was shown to influence arginine hydrolysis obtained with Streptococcus sanguis NCTC 7863 and $S$. milleri 10713.
\end{abstract}

\section{Introduction}

Arginine hydrolysis is a key reaction in the identification of viridans streptococci. In the scheme proposed by Beighton et al. [1], all the species except Streptococcus crista were positive or negative for this reaction, and in the scheme of Kilian et al. [2] only $S$. mitis was reported to have arginine-positive and negative biovars. However, several publications have described discrepancies in arginine hydrolysis results for the same organism when more than one method was used. For example, French et al. [3] reported that 12 strains of $S$. sanguis gave negative results by Cowan's agar method, but positive results with the API20 STREP system. Fertally and Facklam [4] used five methods for arginine hydrolysis and found that all produced some negative results with strains that were expected to be positive. They reported that Niven's method gave positive results with only $13 \%$ of 54 strains expected to be positive. Recently Beighton et al. [5] identified an isolate from the blood of a neutropenic patient as $S$. oralis. It was positive for arginine hydrolysis in the API20 STREP system, but was presumably negative by their method, having been classified as $S$. oralis.

Received 15 April 1996; accepted 30 April 1996.

Corresponding author: Dr P. W. J. West.
There is increasing evidence that viridans streptococci differ in their ecology and pathogenicity $[5,6]$, but if identification includes discrepant results for arginine hydrolysis, organisms will be incorrectly speciated. Most of the clinical isolates in the present study were from neonatal and paediatric blood cultures, routinely identified by the API20 STREP system and Vitek GPI card inoculated and incubated in the Vitek incubatorreader. The two systems often gave different species identities, and differed in individual test results, notably in arginine hydrolysis. Therefore, clinical isolates and reference strains of viridans streptococci were tested for arginine hydrolysis by the two commercial systems, in parallel with three conventional methods, and the effects of inoculum size and growth atmosphere on arginine hydrolysis obtained with $S$. sanguis NCTC 7863 and S. milleri 10713 were investigated.

\section{Materials and methods}

\section{Bacterial strains}

Type strains of $S$. sanguis NCTC 7863, S. mitior NCTC 10712 and $S$. milleri NCTC 10713 were obtained from the National Collection of Type Cultures, 61 Colindale Avenue, London; AR-3, a typical isolate of $S$. oralis, was kindly provided by Prof. D. Beighton. A strain of Enterococcus faecalis consistently positive for arginine 
hydrolysis by all the methods acted as a positive control. Clinical isolates were collected from the blood cultures of neonates, paediatric and adult patients and from urine samples and vaginal swabs submitted to the clinical laboratory of Farwania Hospital, Kuwait; they were stored in skimmed milk $10 \%$ at $-40^{\circ} \mathrm{C}$.

Clinical isolates and control strains were grown on Columbia Agar (Oxoid CM331) with sheep blood 5\%. The plates were incubated anaerobically or in air with $\mathrm{CO}_{2} 10 \%$ for $18-24 \mathrm{~h}$.

\section{Arginine hydrolysis and other biochemical tests}

Clinical isolates and type strains were tested in parallel for arginine hydrolysis by five methods; those described by Waitkins et al. [7] and Facklam and Wilkinson [8], the API20 STREP system, (bioMérieux, Marcy-l'Etoile, France), the Vitek GPI card (bioMérieux Vitek Inc., Hazelwood, MO, USA), and a microtitration plate method. This comprised a medium containing neopeptone (Difco) $1.0 \%$, yeast extract (Oxoid) 0.5\%, L-arginine (BDH 37190) 1\%, glucose $0.05 \%$ and phenol red $0.01 \%$ at $\mathrm{pH} 6.0$. Arginine medium made according to the formula of Waitkins et al. [7] and adjusted to $\mathrm{pH} 7.0$, was dispensed in 2-ml volumes; that of Facklam and Wilkinson [8] was made from Møeller Decarboxylase Base (Difco) in 3-ml volumes in 5-ml screw-topped bottles. All the media were autoclaved at $110^{\circ} \mathrm{C}$ for $10 \mathrm{~min}$. Tests for the presence of a range of enzymes were performed with API20 ZYM galleries [2].

API20 STREP, API20 ZYM galleries and Vitek GPI cards were inoculated according to the manufacturers' instructions. The media of Waitkins et al. and of Facklam and Wilkinson were inoculated with $50 \mu \mathrm{l}$ of the suspension in distilled water (McFarland's standard 4) prepared for the API20 STREP galleries. The inoculum was stabbed into Waitkins' medium with a wire loop, and sterile paraffin oil was dispensed into bottles of the Facklam and Wilkinson medium to a depth of $5 \mathrm{~mm}$. The medium for the microtitration plate method was dispensed in $100-\mu 1$ volumes into 96-well flat-bottomed sterile microtitration plates and inoculated with $100 \mu \mathrm{l}$ of bacterial suspension prepared in broth (neopeptone $1 \%$, yeast extract $0.5 \%$, phenol red $0.01 \%$ ) to an opacity equivalent to
McFarland's standard 4; two drops of sterile paraffin oil were dispensed into the inoculated wells. Vitek cards were put into the Vitek incubator reader at $35^{\circ} \mathrm{C}$, and the other media were incubated at $37^{\circ} \mathrm{C}$ with the lids of the bottled media tightly closed.

Results for the Vitek GPI card were recorded from the laboratory print-out; results for the API ZYM were recorded after incubation for $4 \mathrm{~h}$, those for the API20 STREP at 4 and $24 \mathrm{~h}$ and the microtitration plate method at $24 \mathrm{~h}$. Results for the Waitkins' method and that of Facklam and Wilkinson were read after incubation for 5 days, following the addition of $0.5 \mathrm{ml}$ of Nessler's reagent to the Waitkins' medium.

Dextran production was detected by the production of hard glossy colonies on Tryptone Soya Agar (Oxoid CM131) with sucrose 5\%. Alkaline phosphatase activity was recorded from the API20 STREP and API20 ZYM galleries and N-acetyl- $\beta$-D-glucosaminidase activity from the API20 ZYM [2] gallery.

\section{Results}

Biochemical results for the API20 STREP galleries and Vitek GPI cards were recorded. Isolates were not speciated on the basis of these systems as neither the APILAB API20 STREP (V5.1) nor Vitek GPI computer software identifies viridans streptococci according to current species descriptions [1,2]. Clinical isolates were classified by the scheme of Kilian et al. [2] based on their API20 STREP results for arginine and aesculin hydrolysis, the Voges-Proskauer reaction, the presence of alkaline phosphatase and $\mathrm{N}$-acetyl- $\beta$-D-glucosaminidase and dextran production on sucrose $5 \%$ agar (Table 1). This scheme recognised six species, including both biovars of $S$. mitis. Isolates that did not conform to this classification were not included in the comparative study. NCTC strain 7863 was identified as S. sanguis, NCTC 10712 as $S$. mitis biovar 1, NCTC 10713 as $S$. anginosus and strain AR-3 as $S$. oralis.

Results for arginine hydrolysis by the five methods are shown in Table 2. The API20 STREP, Waitkins' method and the microtitration plate method gave identical results except in the case of one strain of $S$. mitis biovar 1; this was consistently only weakly

Table 1. Speciation of 78 clinical isolates of viridans streptococci according to the scheme of Kilian et al. [2]

\begin{tabular}{|c|c|c|c|c|c|c|c|}
\hline $\begin{array}{l}\text { Biochemical } \\
\text { characteristic }\end{array}$ & S. sanguis & S. gordonii & S. oralis & $\begin{array}{l}S . \text { mitis } \\
\text { biovar } 1\end{array}$ & $\begin{array}{l}S . \text { mitis } \\
\text { biovar } 2\end{array}$ & $S$. anginosus & S. salivarius \\
\hline Arginine hydrolysis & + & + & - & - & + & + & - \\
\hline Aesculin hydrolysis & + & + & - & - & - & d & + \\
\hline Voges-Proskauer reaction & - & - & - & - & - & + & + \\
\hline Dextran production & + & + & + & - & - & - & - \\
\hline Alkaline phosphatase presence & - & + & + & $\mathrm{d}$ & + & + & + \\
\hline $\mathrm{N}$-Acetyl $\beta$-glucosaminidase presence & - & + & + & - & + & d & - \\
\hline Number of strains & 10 & 5 & 16 & 25 & 2 & 13 & 7 \\
\hline
\end{tabular}

+ , Positive result; - , negative result; $\mathrm{d}$, different results obtained with different isolates. 
Table 2. Comparison of arginine hydrolysis by clinical isolates tested by five methods

\begin{tabular}{|c|c|c|c|c|c|c|c|}
\hline \multirow[b]{2}{*}{ Arginine hydrolysis method } & \multicolumn{7}{|c|}{ Percentage of isolates that gave positive results } \\
\hline & $\begin{array}{c}\text { S. sanguis } \\
(10)\end{array}$ & $\begin{array}{c}\text { S. gordonii } \\
\text { (5) }\end{array}$ & $\begin{array}{c}\text { S. oralis } \\
\text { (16) }\end{array}$ & $\begin{array}{c}S . \text { mitis } \\
\text { biovar } 1 \\
(25)\end{array}$ & $\begin{array}{c}S . \text { mitis } \\
\text { biovar } 2 \\
(2)\end{array}$ & $\begin{array}{c}\text { S. anginosus } \\
\text { (13) }\end{array}$ & $\begin{array}{c}\text { S. salivarius } \\
\text { (7) }\end{array}$ \\
\hline API20 STREP & 100 & 100 & 0 & 0 & 100 & 100 & 0 \\
\hline Vitek GPI card & 0 & 0 & 0 & 0 & 0 & 38 & 0 \\
\hline Microtitration plate & 100 & 100 & 0 & 0 & 100 & 100 & 0 \\
\hline Waitkins et al. [7] & 100 & 100 & 0 & 4 & 100 & 92 & 0 \\
\hline Facklam and Wilkinson [8] & 100 & 100 & 0 & 0 & 100 & 54 & 0 \\
\hline
\end{tabular}

( ), Number of isolates tested.

positive in the medium of Waitkins et al. [7], but negative with the other methods used. Strains identified as $S$. sanguis or $S$. gordonii and $S$. mitis biovar 2 gave positive results for arginine hydrolysis by the API20 STREP system and with the media prepared in the laboratory but consistently negative results in the Vitek GPI card. Some isolates of $S$. anginosus were negative for arginine hydrolysis in the Vitek GPI card and in the medium of Facklam and Wilkinson (Table 2). Factors associated with these anomalous results were investigated further.

S. sanguis NCTC 7863 and S. milleri (S. anginosus) NCTC 10713 were always positive for arginine hydrolysis in the API20 STREP system; $S$. mitior ( $S$. mitis) NCTC 10712 and $S$. oralis AR-3 were always negative. This applied both to cultures incubated anaerobically, as recommended by the API20 STREP manufacturers, and in air with $\mathrm{CO}_{2} 10 \%$ as used by French et al. [3]. The intensity of the positive colour in the arginine well after incubation for $24 \mathrm{~h}$ was greater with cultures of strain NCTC 7863 incubated anaerobically, although the result was clearly positive with both methods of incubation. Arginine hydrolysis for NCTC strain 7863 in the Vitek GPI card was invariably negative with cultures grown anaerobically or in $\mathrm{CO}_{2} 10 \%$, even when the inoculum density was increased from 0.5 to 2 McFarland units. Vitek GPI arginine hydrolysis results for clinical isolates of $S$. sanguis, $S$. gordonii and $S$. mitis biovar 2 were negative in identification results printed after incubation for $5-12 \mathrm{~h}$, whereas the $E$. faecalis control was consistently positive after incubation for $4-5 \mathrm{~h}$. The GPI arginine result for $S$. milleri ( $S$. anginosus) NCTC 10713 was positive after incubation for $15 \mathrm{~h}$. Results obtained for arginine hydrolysis with clinical isolates of $S$. anginosus with the Vitek GPI card varied; those printed at $\leqslant 12 \mathrm{~h}$ were usually negative. Repeat testing performed on some isolates of $S$. anginosus showed that although in most cases results were consistent, the same strain could give either a negative or a positive result with the Vitek GPI card. The Vitek GPI card results given in Table 2 are those obtained when the five methods were used in parallel.

The Facklam and Wilkinson method gave negative results with six of 13 isolates of $S$. anginosus when inoculated with $50 \mu \mathrm{l}$ of a McFarland standard 4 suspension, whereas one of 13 was negative by Waitkins' method. Parallel tests with suspensions of S. sanguis NCTC 7863 and S. milleri (S. anginosus) NCTC 10713 in distilled water showed that positive results in the medium of Facklam and Wilkinson required inocula of $c .10^{7} \mathrm{cfu} / \mathrm{ml}$, compared to $c$. $10^{3} \mathrm{cfu} / \mathrm{ml}$ in the Waitkins' medium. The number of viable bacteria inoculated was several times greater than the viable count since Gram-stained smears of the inocula revealed that many of the organisms were still in chains or small clumps.

\section{Discussion}

Reasons why viridans streptococci may give discrepant results in various media used for arginine hydrolysis tests were discussed by Fertally and Facklam [4]. A very important factor, a cause of confusion in the literature for 50 years, is the arginine isomer used. In their original paper, Niven et al. [9] reported the use of D-arginine. However, Barrow and Feltham [10] state that these workers actually used the L-isomer, but incorrectly called it D-arginine because the compound is dextro-rotatory. Fertally and Facklam prepared Niven's medium with the D-isomer of arginine and obtained positive results with only $13 \%$ of 54 strains expected to be positive compared to $94 \%$ positive results obtained with the API20 STREP assay. Kilian et al. [2] reported that they used D-arginine, but their results for the same strains, i.e., NCTC 7863, NCTC 7865 and NCTC 3165 were identical to those of Beighton et al. [1] who used the L-isomer. The package inserts for the API20 STREP and Vitek GPI card do not state which arginine isomer is present in their media.

The inclusion of yeast extract is beneficial in promoting the growth of $S$. sanguis and S. anginosus from small inocula. Yeast extract is present in the Waitkins' formulation, but not in that of Facklam and Wilkinson, who used Møeller decarboxylase broth with arginine $1 \%$. It is notable that in his publication on viridans streptococci, Facklam [11] reported only four of nine type strains of $S$. anginosus-like bacteria ( $S$. anginosus, $S$. constellatus, S. intermedius, Strepto- 
coccus $\mathrm{MG}$ ) positive for arginine hydrolysis. French et al. [3] state that they used 'Cowan's agar method', although they do not give the formula. The agarcontaining medium listed in the appendix of the edition of Cowan's manual [12] cited by French et al. [3] is that of Thornley, which does not contain yeast extract.

Niven's original medium contained glucose at $0.5 \%$ $[9,10]$; however, the formula given by Waitkins et al. [7] did not include glucose, but was otherwise identical to that of Niven except for the inclusion of agar $1 \%$. In the present study, the Waitkins' medium was prepared with and without glucose and, using the inoculum described, no difference was found in arginine hydrolysis results with either control strains or clinical isolates of viridans streptococci.

Also, multiplication of clinical isolates and control strains was observed in the Waitkins' medium, by the production of visible growth after incubation for $24-72 \mathrm{~h}$, even with inocula of $c .10^{3} \mathrm{cfu}$. Visible growth was not always observed in the Facklam and Wilkinson medium, although the rise in $\mathrm{pH}$ associated with arginine hydrolysis occurred within the first $72 \mathrm{~h}$ for strains that produced positive results. Therefore, incubation of these two media for as long as 5 days may be unnecessary. The API20 STREP and phenol red microtitration plate method were inoculated with bacterial populations $>10^{9} \mathrm{cfu}$ and sometimes produced positive results within an incubation time of $6 \mathrm{~h}$.

This work suggests that negative results for arginine hydrolysis with strains of viridans streptococci expected to be positive are a consequence of the use of media that are inadequate for growth, to the metabolism of these organisms from the sizes of inocula used, or to the incubation period employed.

\section{References}

1. Beighton D, Hardie JM, Whiley RA. A scheme for the identification of viridans streptococci. J Med Microbiol 1991; 35: $367-372$.

2. Kilian M, Mikkelsen L, Henrichsen J. Taxonomic study of viridans streptococci; description of Streptococcus gordonii $\mathrm{sp}$. nov. and emended descriptions of Streptococcus sanguis (White and Niven 1946), Streptococcus oralis (Bridge and Sneath 1982) and Streptococcus mitis (Andrewes and Horder 1906). Int J Syst Bacteriol 1989; 39: 471-484.

3. French GL, Talsania H, Charlton JRH, Phillips I. A physiological classification of viridans streptococci by use of the API20STREP system. $J$ Med Microbiol 1989; 28: $275-286$.

4. Fertally SS, Facklam R. Comparison of physiologic tests used to identify non-beta-hemolytic aerococci, enterococci, and streptococci. J Clin Microbiol 1987; 25: 1845-1850.

5. Beighton D, Carr AD, Oppenheim BA. Identification of viridans streptococci associated with bacteraemia in neutropenic cancer patients. $J$ Med Microbiol 1994; 40: 202-204.

6. Frandsen EV, Pedrazolli V, Kilian M. Ecology of viridans streptococci in the oral cavity and pharynx. Oral Microbiol Immunol 1991; 6: 129-133.

7. Waitkins SA, Ball LC, Fraser CAM. A shortened scheme for the identification of indifferent streptococci. J Clin Pathol 1980; 33: 47-52.

8. Facklam RR, Wilkinson HW. The family Streptococcaceae (medical aspects) In: Starr MP, Stolp H, Truber HG, Balows A, Schlegel (eds) The prokaryotes, vol 2. Berlin, Springer-Verlag. 1981: $1572-1597$

9. Niven CF, Smiley KL, Sherman JM. The hydrolysis of arginine by streptococci. J Bacteriol 1942; 43: 651-660.

10. Barrow GI, Feltham RKA (eds). Cowan and Steel's Manual for the identification of medical bacteria, 3rd edn. Cambridge, Cambridge University Press. 1992: 200.

11. Facklam RR. Physiological differentiation of viridans streptococci. J Clin Microbiol 1977; 5: 184-201.

12. Cowan ST. Cowan and Steel's Manual for the identification of medical bacteria, 2nd edn. Cambridge, Cambridge University Press. 1974: 169. 